\title{
Isolated Echogenic Cardiac Focus: Assessing Association with Trisomy 21 by Combining Results from a Prenatal Center with a Bayesian Meta-Analysis
}

\section{(이 (1) $(9)$}

\author{
Authors
}

Elisabeth Wrede ${ }^{1}$, Alexander Johannes Knippel' ${ }^{1}$, Pablo Emilio Verde ${ }^{2}$, Ruediger Hammer ${ }^{1}$, Peter Kozlowski ${ }^{1}$

\section{ABSTRACT} group.
Objective To investigate the clinical relevance of an isolated echogenic cardiac focus (iECF) as a marker for trisomy 21 using a large second-trimester collective including a low-risk sub-

Materials and Methods We retrospectively evaluated 125211 pregnancies from 2000-2016 and analyzed all iECF cases with regard to chromosomal anomalies. It consisted of an early second-trimester collective from $14+0-17+6$ weeks $(n=34791)$ and a second-trimester anomaly scan collective from $18+$ $0-21+6$ weeks. Two a priori risk subgroups (high and low risk) of the latter were built based on maternal age and previous screening test results using a cut-off of 1:300. Likelihood ratios (LR) of iECF for the detection of trisomy 21 , trisomy 13 , trisomy 18 and structural chromosomal anomalies were estimated.

Results In total, 104001 patients were included. An iECF was found in 4416 of 102847 euploid fetuses (4.29\%) and in 64 of 557 cases with trisomy 21 (11.49\%) giving a positive LR of 2.68 (Cl: $2.12-3.2)$. The sensitivity was $11.5 \%$ at a false-positive rate of $4.29 \%$ (Cl:4.17-4.42) with $\mathrm{p} \leq 0.01 \%$. In the high-and lowrisk subgroups, the prevalence of iECF was comparable: $5.08 \%$ vs. $5.05 \%$. The frequency of trisomy 21 was $0.39 \%, 98 / 24979$ vs $0.16 \%, 69 / 44103$. LR + was $3.86(2.43-5.14)$ and $2.59(1.05-$ 4). For both subgroups the association of iECF with trisomy 21 was statistically significant. The prevalence of structural chromosomal anomalies in the second-trimester anomaly scan collective was $0.08 \%$ (52/68967), of which 2 showed an iECF. Conclusion The detection of an iECF at the time of $14+0-21+6$ weeks significantly increases the risk for trisomy 21 in the highrisk and in the low-risk subgroups and does not statistically change the risks for trisomy $13 / 18$ or structural abnormalitie.

\section{Introduction}

Most expectant parents want to know as early as possible whether their unborn child will be born healthy or with a structural malformation. Particularly with regard to chromosomal disorders, couples wish to have early diagnostic clarification in order to consider adjustments needed in the event of giving birth to a child with abnormalities or to terminate the pregnancy. Trisomy 21 is the most common chromosomal abnormality in liveborn infants with an in- cidence of $1 / 600-1 / 800$ in the general population [1]. First Trimester Screening (FTS) at 11+0-13+6 weeks of pregnancy offers an early assessment of the risk for aneuploidies. Nuchal translucency and other ultrasound parameters in combination with maternal age and biochemical parameters, like free beta HCG and PAPP-A, can be used to estimate an individualized risk for the three most common chromosomal disorders (trisomy 21, 13 and 18) at an early stage [2-4]. With a detection rate of $90 \%$ and a false-positive 
rate of $5 \%$, this is a very sensitive screening method for detecting trisomies. In the second-trimester anomaly scan, markers can be used to adjust the risk for trisomy 21 . Agaard-Tillery et al. published a study with 7842 pregnant women at the second-trimester ultrasound scan [5].They demonstrated that the detection rate of trisomy 21 can increase from 93-98\% if the basal risk from first-trimester screening was modified with the marker screening result of the second-trimester ultrasound scan by considering the positive and negative likelihood ratios in the presence or absence of these markers. Considering this fact, the detection of second-trimester ultrasound markers may lead to an increased number of diagnostic procedures [6, 7]. Regarding all second trimester markers, the echogenic cardiac focus (ECF) is the most controversial [8] because it is the most prevalent marker among the normal population with a prevalence of approximately $5-10 \%$ in a normal second-trimester collective [9]. The vast majority of studies showed a significantly increased risk for trisomy 21 if the echogenic focus occurs in combination with other minor markers [10-12]. There is controversy as to whether the echogenic focus, if it occurs in isolation, also increases the risk for trisomy 21 and in particular whether this increase in risk also exists in an unselected normal population. Furthermore, many past studies have suggested an isolated echogenic cardiac focus (iECF) to be associated with an increased risk of trisomy in high-risk populations, e. g. due to advanced maternal age or increased risk of previous screening [13-16]. Other publications reported a tendency towards an increased risk also in low-risk pregnancies, but failed to show a statistically significant result $[5,14,17-19]$. This may also be due to an insufficient number of cases in the individual low-risk studies and the associated low statistical power corresponding to the lower prevalence of trisomy 21 in these low-risk studies. Furthermore, observational databases are known to suffer from a series of internal validity biases [20]. Therefore, a direct interpretation of results at face value could be misleading. The aim of this study is to assess the clinical relevance of the iECF by combining the data from our prenatal center with a Bayesian meta-analysis. We also formed a low-risk subgroup in order to provide information on how to counsel parents in pregnancies with an iECF. Our aim was to clarify three questions: First, does the discovery of an isolated echogenic cardiac focus with an otherwise completely normal ultrasound result change the patient's risk to such an extent that this finding must be included in prenatal counselling? Second, does an iECF increase the risk for trisomy 21 in the low-risk group with regard to the low prevalence of this disease? Noninvasive prenatal testing (NIPT) is the analysis of cell-free DNA from maternal blood with a high negative predictive value, which makes it an option for the clarification especially of medium-risk cases. However, this method does not cover the same spectrum of anomalies as classic invasive diagnostic testing, for example structural chromosomal anomalies are not addressed by the current NIPT tests. Thus, our third question is: Is there any evidence of other chromosomal abnormalities associated with iECF that would indicate that a cell-free DNA test is not sufficient for clarification of the iECF?

\section{Methods}

This was a retrospective cohort study of ultrasound examinations in a tertiary referral center that included all singleton pregnancies between $14+0$ and $21+6$ weeks in the years 2000-2016 $(n=125211)$. In addition, we performed a subgroup analysis by dividing the second-trimester anomaly scan collective $18+0$ to $21+6$ weeks into two a priori risk groups based on the maternal age and, if available, previous screening test results and defined a risk cutoff point of $1 / 300$. As a priori high risk we rated: a) maternal age 35 or older - no screening test, b) age 35 or older with a risk cut-off $\geq 1: 300$, and c) younger than 35 but a risk cut-off $\geq 1: 300$. As a priori low risk we rated d) younger than 35 with no test, e) younger than 35 with a risk cut-off $<1: 300$, and $f$ ) age 35 or older but a risk cut-off<1:300. We used high-resolution ultrasound equipment (Toshiba Aplio 500, GE Voluson 730, E8, E10). Ultrasound examinations were performed by DEGUM II certified specialists in obstetric ultrasound with several years of special experience in prenatal medicine. We obtained written informed consent from participants and the Ethics Committee (study number 5588) of the University of Düsseldorf accepted the study. Information on any fetal chromosomal abnormalities was either taken from prenatal cytogenetic findings or, if not available, from the requested postnatal $\mathrm{U} 1$ reports. Neonates with normal phenotypes were assumed to have normal karyotypes. Any postnatal phenotypic suspicion of a chromosomal disorder was cytogenetically clarified. We reviewed our ultrasound database for any entry regarding an echogenic heart focus. In each identified case, the ultrasound findings were evaluated for any further abnormality to identify the isolated cases of ECF. The ECF was defined as an echo-rich structure in or next to the papillary muscle of the right and/or left ventricle that corresponded to the brightness of bones. We classified an echogenic cardiac focus as "isolated" (iECF) if there were no further malformations, markers or any other clinically relevant abnormalities. All cases with known fetal karyotype before examination ( $n=1586 ; 1.27 \%$ ) were excluded. We also excluded cases with aneuploidies other than 13 , 18 or 21 ( $n=197 ; 0.16 \%$ ), all cases without written consent to anonymous study participation $(n=11 ; 0.01 \%)$ and all cases "lost to follow up" in which neither the karyotype nor the postnatal examination findings were clearly known ( $n=19416 ; 15.51 \%)$. An overview is presented in > Table 1. Fetuses with known euploid karyotype or missing stigmata of aneuploidy at birth were classified as "euploid". In terms of structural anomalies, we did not distinguish between unbalanced and balanced findings and we also assigned the microdeletions to this group. $>$ Table $\mathbf{2}$ gives an overview of study exclusions and karyotypes. After completion of the classification, we constructed $2 \times 2$ tables to calculate the proportion of isolated ECFs among the chromosomally abnormal and the euploid fetuses. Likelihood ratio was calculated as a quotient of iECF prevalence among the aneuploid cases divided by the corresponding prevalence among the euploid cases.

\section{Meta-analysis}

For the meta-analysis we investigated all studies that aimed to estimate the population prevalence of isolated ECF and the associated risk of trisomy 21 in a coherent collective between 1998-01-01 and 2019-08-01. For this purpose we analyzed all publications used in the meta-analysis of Agathokleous et al. 2013 [21] based 
- Table 1 Study exclusions and characteristics of the included patients.

\begin{tabular}{|l|l|}
\hline $\begin{array}{l}\text { Causes for the study exclusion/ } \\
\text { characteristics }\end{array}$ & Number/value \\
\hline Total number of patients & 125211 \\
\hline Exclusions & \\
\hline Aneuploidies other than Trisomies $13,18,21$ & $197 / 125211(0.16 \%)$ \\
\hline Invasive diagnosis before ultrasound & $1586 / 125211(1.27 \%)$ \\
\hline No study consent & $11 / 125211(0.01 \%)$ \\
\hline No outcome & $19416 / 125211(15.51 \%)$ \\
\hline Total exclusions & $21210 / 125211(1694 \%)$ \\
\hline Included & $\mathbf{1 0 4 0 0 1 / 1 2 5 2 1 1}(\mathbf{8 3 0 6} \%)$ \\
\hline Mean GA & 18.88 \\
\hline Number GA group '14+0 to $17+6{ }^{\prime}$ & $34791(33.45 \%)$ \\
\hline Number GA group '18+0 to $21+6{ }^{\prime}$ & $69210(66.55 \%)$ \\
\hline Mean maternal age during examination & 33.96 \\
\hline Age $\geq 35$ years & $50600 / 104001(48.65 \%)$ \\
\hline Age<35 years & $53401 / 104001(51.35 \%)$ \\
\hline Mean maternal age group '14+0 to $17+6{ }^{\prime}$ & 36.35 \\
\hline Mean maternal age group '18+0 to $21+6{ }^{\prime}$ & 32.77 \\
\hline Ethnicity & $103552(99.57 \%)$ \\
\hline Caucasian & $106(0.1 \%)$ \\
\hline Asian & $75(0.07 \%)$ \\
\hline Oriental & $75(0.07 \%)$ \\
\hline Black & $193(0.19 \%)$ \\
\hline Mixed & \\
\hline
\end{tabular}

Table 2 Karyotypes of excluded and included cases.

\begin{tabular}{|l|l|l|}
\hline Karyotype & $\begin{array}{l}\text { Number of } \\
\text { included cases } \\
\text { (percent) }\end{array}$ & $\begin{array}{l}\text { Number of } \\
\text { excluded cases } \\
\text { (percent) }\end{array}$ \\
\hline Euploid & $102847(82.14 \%)$ & $2135(1.71 \%)$ \\
\hline Trisomy $\mathbf{2 1}$ & $557(0.44 \%)$ & $69(0.06 \%)$ \\
\hline $\begin{array}{l}\text { Structural chromosomal } \\
\text { anomalies }\end{array}$ & $431(0.34 \%)$ & $80(0.06 \%)$ \\
\hline Trisomy $\mathbf{1 8}$ & $120(0.1 \%)$ & $16(0.01 \%)$ \\
\hline Trisomy 13 & $46(0.04 \%)$ & $(0 \%)$ \\
\hline No outcome & $(0 \%)$ & $18661(14.9 \%)$ \\
\hline Triploidy & $(0 \%)$ & $58(0.05 \%)$ \\
\hline Other aneuploidy & $(0 \%)$ & $11(0.01 \%)$ \\
\hline Gonosomal aneuploidy & $(0 \%)$ & $180(0.14 \%)$ \\
\hline Total & $\mathbf{1 0 4 0 0 1}(\mathbf{8 3 0 6} \%)$ & $\mathbf{2 1 2 1 0 ( 1 6 . 9 4 \% )}$ \\
\hline * unbalanced, balanced and microdeletions. & \\
\hline
\end{tabular}

- Fig. 1 Selection of literature for the meta-analysis.

on the Supplemental list ' $>$ Table 1S'. We completed this list of studies for the time from 2010 onwards based on a structured query in PubMed for the echogenic cardiac focus: (( ("echogenic focus" OR "echogenic foci")) OR ("hyperechoic focus" OR "hyperechoic foci")) OR ("echogenic cardiac focus" OR "echogenic cardiac foci")) OR ("echogenic heart focus" OR "echogenic heart foci")) OR ("echogenic intracardiac focus" OR "echogenic intracardiac foci")) OR "golf ball”) AND ("2010/01/01" [Date - Publication]: "2019/08/01" [Date - Publication]). From the results of this query (267 articles in English were found), we then excluded non-medical studies, studies in uncommon languages, studies not focusing on the ECF as a soft marker for trisomy 21, reviews/editorials/meta-analysis and overlapping papers for 2010 that were already assessed by Agathokleous. In total, 19 studies from the Agathokleous list dealt with the subject of ECF plus 14 were left over from our query after primary exclusions. Among these 33 studies, we classified publications as eligible for our meta-analysis, if: 1) A $2 \times 2$ cross table could be extracted for the incidence of isolated ECF in both euploid and trisomy 21 fetuses. 2) Study design: prospective or retrospective cohort studies. 3) No case control studies and case reports. 4) Classifying the risk characteristic of the study cohort concerning trisomy 21 was possible. 5) The procedure for collecting outcomes regarding trisomy 21 for the whole collective must be described. 6) The number of exclusions lost to follow-up was given. 7) Gestational age at examination was between $14+0$ and $26+6$ weeks (overview in > Fig. $\mathbf{1}$ ).

From the eligible studies we extracted the number of true positives, true negatives, false positives and false negatives. We then classified them as 'high risk' or 'normal/low risk' and 'prospective' or 'retrospective' according to the indications in the paper. The included studies and the $2 \times 2$ table values for isolated ECF are displayed in $>$ Table 3. LR + was calculated on the basis of these numbers.

\section{Statistical methods}

In this study, we used a meta-analysis of previously published studies, with diagnostic test accuracy of the iECF marker to build a bias 
- Table 3 Studies included in the meta-analysis.

\begin{tabular}{|c|c|c|c|c|c|c|c|c|c|}
\hline Author & Year & Population & Design & TP & FP & FN & TN & Total & $\mathrm{LR}+$ calculated \\
\hline Manning [24] & 1998 & high risk & $\mathrm{p}$ & 2 & 21 & 15 & 863 & 901 & 4.95 \\
\hline Sohl [25] & 1999 & high risk & $\mathrm{p}$ & 12 & 151 & 33 & 2488 & 2684 & 4.66 \\
\hline Thilaganathan [26] & 1999 & high risk & $\mathrm{p}$ & 0 & 143 & 10 & 16763 & 16916 & 0 \\
\hline Wax [27] & 2000 & high risk & $\mathrm{p}$ & 2 & 21 & 5 & 751 & 779 & 10.5 \\
\hline Winter & 2000 & high risk & $\mathrm{p}$ & 5 & 130 & 21 & 2689 & 2845 & 4.17 \\
\hline Prefumo [28] & 2001 & low risk & $r$ & 0 & 239 & 6 & 7443 & 7688 & 0 \\
\hline Huggon [29] & 2001 & high risk & $\mathrm{p}$ & 5 & 543 & 75 & 6361 & 6984 & 0.79 \\
\hline Coco [30] & 2004 & low risk & $\mathrm{p}$ & 1 & 432 & 10 & 12229 & 12672 & 2.66 \\
\hline Lamont [31] & 2004 & low risk & $r$ & 1 & 310 & 13 & 10445 & 10769 & 2.48 \\
\hline Smith-Bindman [32] & 2007 & high risk & $\mathrm{P}$ & 15 & 211 & 230 & 8496 & 8952 & 2.53 \\
\hline Weisz [17] & 2007 & low risk & $r$ & 1 & 88 & 11 & 2232 & 2332 & 2.2 \\
\hline Shanks & 2009 & low risk & $r$ & 14 & 1998 & 204 & 59895 & 62111 & 1.99 \\
\hline Huang & 2010 & low risk & $\mathrm{p}$ & 2 & 209 & 23 & 6884 & 7118 & 2.72 \\
\hline Hurt [33] & 2016 & low risk & $\mathrm{p}$ & 3 & 600 & 28 & 18210 & 18841 & 3.03 \\
\hline Ginsberg [34] & 2017 & low risk & $r$ & 20 & 1340 & 42 & 19270 & 20672 & 4.96 \\
\hline Total & & & & 83 & 6436 & 726 & 175019 & 182264 & \\
\hline
\end{tabular}

correction model for the diagnostic results of our prenatal database. Using the $2 \times 2$ tables of published diagnostic results, we performed a multi-parameter Bayesian meta-analysis of the sensitivities and specificities. The posterior distributions of the marginal pooled sensitivity and specificity were used as meta-analytic priors to adjust the results of the prenatal database. This adjustment was performed on the sensitivities and specificities of the prenatal database and by handling the LR + and LR- as functional parameters. Therefore, the Bayesian computations were performed at the level of sensitivity and specificity and results are transformed on the scale of LR + and LR-.

The studies included in the meta-analysis suffer from a series of uncontrolled variabilities, e. g., different internal quality, different study design, variation in the study population and diagnostic settings. Those sources of variation are non-systematic resulting in a complex random heterogeneity between studies. In addition, the number of studies included in the meta-analysis is small $(n=15)$. Therefore, a specially designed Bayesian method has to be used to make a meta-analysis of this kind of data. In this study, we applied the meta-analysis model based on random effects with scale mixtures of normal distributions implemented in the R's package bamdit (Bayesian Meta-Analysis of Diagnostic Test Data). The results of the meta-analysis model are displayed by plotting the observed TPRs (True-Positive Rates) versus the FPRs (False-Positive Rates). The Bayesian model is summarized by the 50,75 and $95 \%$ posterior predictive curves. In addition, we displayed 500 model's prediction of the combination of TPRs and FPRs.

\section{Statistical computations}

The statistical analysis was performed with the statistical software $R$ version 3.5.2 ( $R$ Core Team, 2019). The Bayesian meta-analysis of diagnostic test accuracy was performed with $R$ package bamdit [22]. Statistical analysis was performed with the statistical software $R$ version 3.5.2 (R Core Team, 2019). Bayesian models are not analytically tractable. Estimation of posterior probabilities was based on MCMC (Markov Chain Monte Carlo) computations. In each analysis, we used two MCMC runs of 20000 iterations and we discarded the first 5000 for the burn-in period. Convergence was assessed visually using the R package coda. The results of the Bayesian analyses are presented as posterior distributions and their summaries: Posterior means, standard deviations, quantiles $(2.5,50$, and $97.5 \%$ and the histogram of the posteriors.

\section{Results}

A total number of 125211 patients with a singleton pregnancy between $14+0$ and $21+6$ weeks underwent prenatal ultrasound examination during the study period. See $>$ Table $\mathbf{1}$ for more details and causes for study exclusion. An overview of the karyotypes of excluded and included cases is given in $>$ Table 2 . The overall prevalence of isolated echogenic foci in the current study population was $4.33 \%(4480 / 104001)$. In total, an isolated ECF was found in 4416 of 102847 euploid fetuses (4.29\%) and in 64 of 557 cases with trisomy $21(11.49 \%)$ which led to a positive likelihood ratio $(L R+)$ of 2.68 (Cl: 2.12-3.2) for the entire study population ( $\bullet$ Table 4 ).

Subsequently, we divided the $18+0-21+6$ second-trimester anomaly scan group into two subgroups, high and low risk for fetal trisomy 21 . The prevalence of iECF was very similar in both groups 
- Table 4 Results of the meta-analysis.

\begin{tabular}{|c|c|c|c|c|c|c|c|c|c|c|}
\hline Type of data & Population & LR+ & LR- & TP & FP & FN & TN & Total & Sens. & Spec. \\
\hline Our center & mixed & $2.68(2.12-3.20)$ & 0.92 & 64 & 4416 & 493 & 98431 & 103404 & 0.12 & 0.96 \\
\hline Our center & high risk & $3.86(2.43-5.14)$ & 0.85 & 19 & 1251 & 79 & 23630 & 24979 & 0.19 & 0.95 \\
\hline Our center & low risk & $2.59(1.05-4.00)$ & 0.92 & 9 & 2219 & 60 & 41815 & 44103 & 0.13 & 0.95 \\
\hline Meta-analysis & $\begin{array}{l}\text { posterior mean } \\
\text { (posterior } 95 \% \text { interval) }\end{array}$ & $3.11(1.84-4.92)$ & 0.93 & & & & & & 0.11 & 0.97 \\
\hline $\begin{array}{l}\text { MA/our center } \\
\text { combined }\end{array}$ & mixed & $2.65(2.11-3.3)$ & $0.93(0.90-0.94)$ & & & & & & & \\
\hline $\begin{array}{l}\text { MA/our center } \\
\text { combined }\end{array}$ & high risk & $2.92(2.05-3.90)$ & $0.90(0.85-0.96)$ & & & & & & & \\
\hline $\begin{array}{l}\text { MA/our center } \\
\text { combined }\end{array}$ & low risk & $2.33(1.51-3.30)$ & $0.93(0.88-0.97)$ & & & & & & & \\
\hline
\end{tabular}

Posterior $L R+$ of the meta-analysis $(M A)$ and combined $L R+$ of our center + meta-analysis. $T P=$ true positives, $F P=$ false positives, $F N=f a l s e$ negatives, $\mathrm{TN}=$ true negatives, sens. $=$ sensitivity, spec. $=$ specificity.

with $5.08 \%$ (1270/24 979) in the high-risk subgroup and 5.05\% (2228/44.103) in the low-risk subgroup. As expected, the frequency of trisomy 21 was higher in the first subgroup than in the latter $(0.39 \%, 98 / 24979$ vs. $0.16 \%, 69 / 44103)$. Overall for our centerspecific collective these numbers led to better screening performance of the iECF in the high-risk group compared to the low-risk group with a sensitivity of $19.39 \%(\mathrm{Cl}: 11.56-27.21)$ vs. $13.04 \%$ (Cl: 5.1-20.99) at an almost identical FPR of $5.03 \%(\mathrm{Cl}: 4.76-5.3)$ vs. $5.04 \%$ (Cl: 4.83-5.24). The LR + was calculated as 3.86 (Cl: $2.43-$ $5.14)$ in the high-risk group and 2.59 (Cl: 1.05-4) in the low-risk group ( $\triangleright$ Table 4 ).

The ECF in combination with one or more other markers showed a clearly higher $L R+(31.9)$ than the iECF. The overall consideration of other markers in isolation resulted in a slightly higher $L R+(4.39)$. The highest $L R+(88.9)$ was found when two or more other markers than ECF were diagnosed in combination.

\section{Results of the meta-analysis}

The meta-analysis included 15 studies with a total of 182264 patients. After combining all the data from the included studies, the posterior mean LR + was calculated as 3.11 and the posterior $95 \%$ confidence interval ranged from 1.84-4.92 ( $\vee$ Table 4 ). The pooled sensitivity of 1.11 and the specificity of 0.97 were used as meta-analytic priors to adjust the results of the prenatal database. After the adjustment, the combined (meta-analysis and our database) mean $\mathrm{LR}+$ for the total/mixed collective almost did not change $(2.65(\mathrm{Cl}$ : 2.11-3.3)). In both the high-risk and the low-risk subgroup, the mean LR + decreased to 2.92 and 2.33 (high-/low-risk) and the $95 \%$ confidence intervals noticeably narrowed to $2.05-3.90$ and $1.51-$ 3.30 ( $\vee$ Table 4). $\triangleright$ Fig. 2 shows the results of the meta-analysis and gives the joint probability distribution. In order to better display the results, we plotted the false-positive rate (1-specificity) between 0 and 0.25 , and the true-positive rate (sensitivity) is displayed between 0 and 0.5 . The area within the lines predicts the region where we expect the results of an unknown new study. The outer line represents the $90 \%$ posterior interval, and the next 2 lines represent the 75 and $50 \%$ posterior interval.( $>$ Table 1S).

\section{Occurrence of trisomy 13,18 and structural anomalies with echogenic foci}

In the entire study group we found no case of trisomy 18 with an isolated ECF. All 120 cases showed further severe malformations or multiple markers of aneuploidy. For trisomy 13 we found 2 out of 46 cases with ECF and an otherwise completely normal detailed ultrasound result. The first case was referred at $16+0$ weeks of gestation primarily for invasive diagnostic testing because of high maternal age ( 44.8 years). In the ultrasound examination we found no further anatomical abnormalities except an ECF in the left ventricle. Amniocentesis revealed a mosaic trisomy 13 with the karyotype $47, X Y,+13 / 46$, XY, the child was born alive with $2160 \mathrm{~g}$ without phenotypic abnormalities. In the second case we detected two ECFs, one in the left and one in the right ventricle, in primary invasive testing in a 34.8-year-old patient at $14+5$ weeks. The crown rump length of the fetus corresponded to $13+6$ weeks. No further ultrasound abnormalities were found. Chromosomal analysis in all examined metaphases from two independent amniotic fluid cultures revealed the karyotype $47, X Y+13$. The mother decided to terminate the pregnancy. No autopsy was performed. From these figures, an LR + of iECF for trisomy 13 of 1.01 was calculated $(\mathrm{Cl}$ : $0-2.32$ ). The prevalence of structural chromosomal anomalies in the second-trimester anomaly scan collective was $0.08 \%$ (52/68 967), of which 2 showed an iECF. This resulted in a LR + of 0.76 at a $95 \% \mathrm{Cl}$ of $0-1.75$. There was no association between structural chromosomal anomalies and iECF.

\section{Discussion}

The results of our study support the conclusion that an isolated echogenic cardiac focus also in otherwise inconspicuous ultrasound examinations increases the a priori risk by a factor that is with $95 \%$ probability greater than 1.5 . Based on the individual a priori risk, 


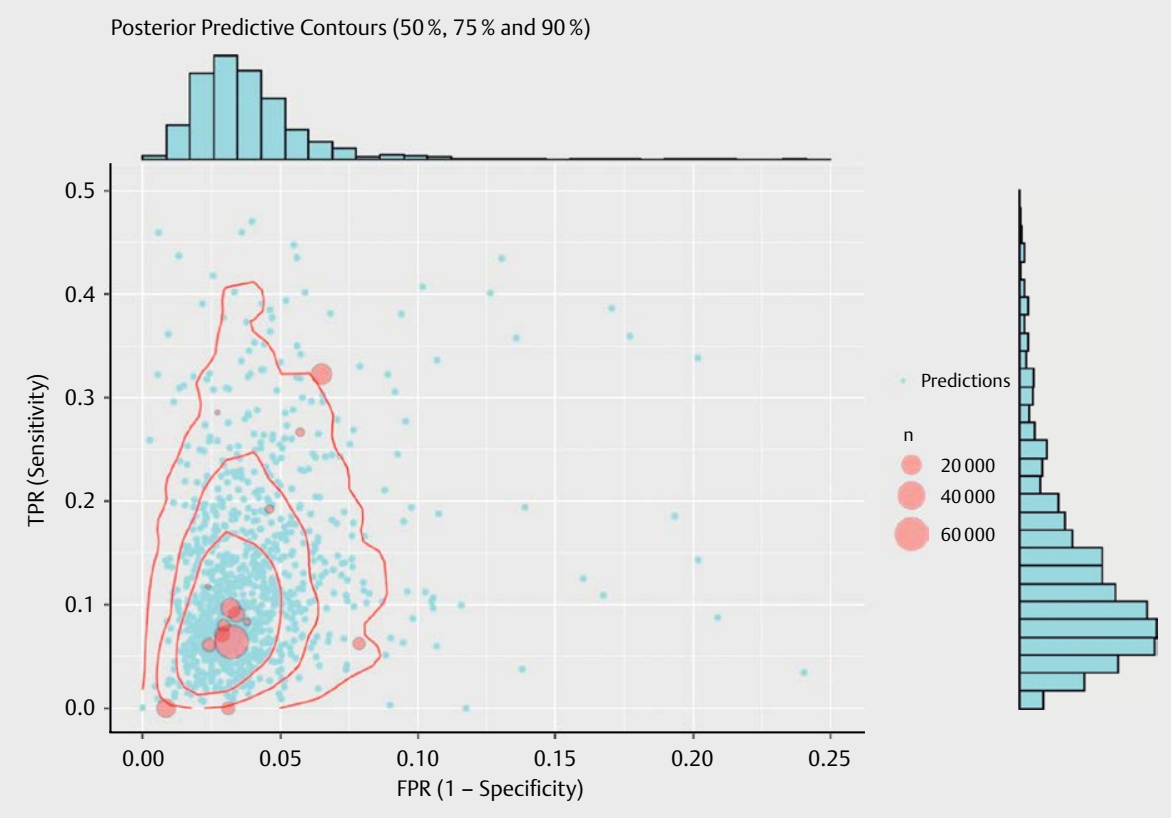

Fig. 2 Zoom of the results of the meta-analysis: Data and predictive posterior contours.

this risk increase regarding trisomy 21 applies to both high-risk and low risk pregnancies and should be taken into account in prenatal counselling. By combining the data from our center with the results of the meta-analysis, the mean LR of the high-risk group converges substantially with the mean LR of the low-risk group and the mean $L R$ of the total mixed population. The $95 \%$ posterior intervals also overlap clearly. Assuming that the pre-selection is stronger in the high-risk group and the investigator's expectations focus more on a possible trisomy 21, which may favor the detection of an ECF, this approximation of mean LRs supports the assumption that the effective likelihood ratio for isolated ECF is not markedly dependent on a priori risk. Furthermore, we found no evidence of association with iECF and structural chromosomal anomalies in our data. Overall, the prevalence of iECF and trisomy 21 as well as the distribution of pregnancy weeks in our study are essentially consistent with previous comparable reports [5, 14, 17-19]. However, these figures must also be seen in the context of a large number of publications on second-trimester risk calculation for soft marker screening. In 2001, Nyberg et al. published a statistically significant association with an LR + of $6.8 \%$ if isolated ECF was found without a systematic search for other markers and of 1.8 (Cl: $1.0-3.2)$ if all other markers were systematically excluded [10]. Our results are consistent with this publication. Agathokleous and Nicolaides came to a slightly different conclusion in their 2013 meta-analysis in which they derived the $L R+$ for isolated ECF by multiplying the pooled LR + for ECF (5.83, Cl: 5.02-6.77) by the negative LR of each other marker [21]. The calculated LR + of an isolated ECF in this study was 0.95 , which conflicts with our observations. However, Nyberg et al. evaluated only 6 soft markers (nuchal thickening, hyperechoic bowel, short humerus, short femur, pyelectasis and ECF), while Agathokleous additionally included ventriculomegaly, ARSA (aberrant right subclavian artery) and present or absent nasal bone.
In particular, the detection of ARSA with its high LR + of $21.48(\mathrm{Cl}$ : 11.48-40.1) is indispensably connected to the use of high-resolution ultrasound techniques Such fluctuations confirm our conviction that, for genetic counselling, likelihood ratios should be derived from high-quality meta-analyses and not from single-center publications. The main strength of our study is the high number of cases which allowed the establishment of a low-risk subgroup with sufficient statistical power to test the association of iECF and trisomy 21. A further strength is the use of a special method for combining evidence from different publications with the data of a coherent collective. Thus, we were able to minimize the bias by uncontrolled variability between different examination settings and populations.

One weakness of our study is a possible non-response bias by the exclusion of $15 \%$ of cases in which the definitive outcome of pregnancy could not be determined. If we assume that parents or the referring gynecologists tend to inform the prenatal medicine unit probably more frequently in cases of abnormal outcome, the exclusion of all non-responders would increase the LR + of iECF for trisomy 21 , if a disproportionate number of families with trisomy 21 and iECF reported back (true positives). If, however, we assume that in the $15 \%$ with unknown outcome there is no case of trisomy 21 and distribute these cases between the true negatives and the false positives according to the prevalence of iECF of about $5 \%$, the $\mathrm{LR}+$ would only change slightly from 2.68 to 2.67 for our general collective. However, we can only speculate on the number of children with Down's syndrome that are concealed in the $15 \%$ figure without outcome. Another weakness is that an inconspicuous phenotype at birth does not exclude chromosomal trisomy 21 . This may underestimate the number of Down's syndrome cases detected postnatally. Karyotyping of all included cases would certainly be the gold standard but we think that this is very difficult to achieve 
for such a large coherent collective. Furthermore, phenotypically normal children could have a cardiac defect that is not immediately noticeable after birth. Therefore, a potential shortcoming of the term 'isolated ECF' should be mentioned. Another inherent weakness of our study is the general preselection of patients, who are referred to a prenatal center, hence having an elevated risk for trisomy 21 . In addition to e. g. maternal age, family genetic predispositions, drug or radiation exposure, related marriage and a variety of smaller and larger ultrasound abnormalities become important for the referring gynecologist. Thus, our figures cannot be representative for an unselected normal collective, even after including evidence from different studies. Assignment to the "lowrisk" group for trisomy 21 was made only on the basis of maternal age or previous FTS findings. Only a few studies assessed an increased risk of trisomy 18 , trisomy 13 or structural abnormalities based on an isolated echogenic focus [23-25].

With respect to trisomy 13 and 18 , we found no reasonable use for the ECF as a marker. In summary, finding of an isolated echogenic heart focus presented significant associations with Down syndrome among pregnant women in both high- and low-risk groups. The individual risk burden of each patient should be determined and discussed as a part of genetic counselling. Since 2012 a new assessment tool for chromosomal abnormalities, especially with regard to trisomy 21 , has been available. Noninvasive prenatal testing (NIPT) is able to detect placental cell-free DNA fragments in maternal blood. The NIPT test is a useful variant for clarifying patients with a medium-risk constellation [26]. The limitations of the NIPT are a lack of feasibility in $5 \%$ of cases due to an insufficient concentration of placental DNA in the maternal plasma, as well as discordant findings between NIPT and genetic analysis by placental mosaicism [27]. Despite the high detection rate for trisomy 21, it should be emphasized that NIPT is not regarded as a diagnostic procedure, but as a screening test like FTS. In the case of high-risk constellations, we consider diagnostic procedures by an experienced examiner to be the better alternative. Regarding the risks of amniocentesis, a 2015 meta-analysis concludes that the combined procedural risk of miscarriage for amniocentesis is $0.11 \%(95 \% \mathrm{Cl}$ : $-0.04 \%$ to $0.26 \%$ ) [28]. In daily practice, genetic counselling becomes more and more complex due to the increasingly refined ultrasound techniques and sophisticated screening and diagnostic capabilities. It is essential for an expectant mother and her family to undergo in-depth counselling exploring all options.

\section{Conflict of Interest}

The authors declare that they have no conflict of interest.

\section{References}

[1] Thompson MMR, Willard H. Genetics in Medicine. $5^{\text {th }}$ ed. Philadelphia: Thompson and Thompson; 1991

[2] Nicolaides KH, Azar G, Byrne D et al. Fetal nuchal translucency: Ultrasound screening for chromosomal defects in first trimester of pregnancy. BMJ 1992; 304: 867-869
[3] Alldred SK, Takwoingi Y, Guo B et al. First trimester ultrasound tests alone or in combination with first trimester serum tests for Down's syndrome screening. The Cochrane Database of Systematic Reviews 2017; 3: Cd012600

[4] Audibert F, Dommergues M, Benattar C et al. Screening for Down syndrome using first-trimester ultrasound and second-trimester maternal serum markers in a low-risk population: A prospective longitudinal study. Ultrasound in obstetrics \& gynecology: The Official Journal of the International Society of Ultrasound in Obstetrics and Gynecology 2001; 18: 26-31

[5] Aagaard-Tillery KM, Malone FD, Nyberg DA et al. Role of second-trimester genetic sonography after Down syndrome screening. Obstetrics and Gynecology 2009; 114: 1189-1196

[6] Benacerraf BR. Should sonographic screening for fetal Down syndrome be applied to low risk women? Ultrasound in obstetrics \& gynecology: The Official Journal of the International Society of Ultrasound in Obstetrics and Gynecology 2000; 15: 451-455

[7] Doubilet PM, Copel JA, Benson CB et al. Choroid plexus cyst and echogenic intracardiac focus in women at low risk for chromosomal anomalies: The obligation to inform the mother. Journal of Ultrasound in Medicine: Official Journal of the American Institute of Ultrasound in Medicine 2004; 23: 883-885

[8] Rodriguez R, Herrero B, Bartha JL. The continuing enigma of the fetal echogenic intracardiac focus in prenatal ultrasound. Current Opinion in Obstetrics \& Gynecology 2013; 25: 145-151

[9] Bromley B, Lieberman E, Laboda L et al. Echogenic intracardiac focus: A sonographic sign for fetal Down syndrome. Obstetrics and Gynecology 1995; 86: 998-1001

[10] Nyberg DA, Souter VL, El-Bastawissi A et al. Isolated sonographic markers for detection of fetal Down syndrome in the second trimester of pregnancy. Journal of Ultrasound in Medicine: Official Journal of the American Institute of Ultrasound in Medicine 2001; 20: 1053-1063

[11] Nicolaides KH, Wegrzyn P. [Sonographic features of chromosomal defects in the second trimester of pregnancy]. Ginekologia polska 2005; 76: 528-535

[12] Vintzileos AM, Egan JF. Adjusting the risk for trisomy 21 on the basis of second-trimester ultrasonography. American Journal of Obstetrics and Gynecology 1995; 172: 837-844

[13] Winter TC, Anderson AM, Cheng EY et al. Echogenic intracardiac focus in 2nd-trimester fetuses with trisomy 21: Usefulness as a US marker. Radiology 2000; 216: 450-456

[14] Anderson N, Jyoti R. Relationship of isolated fetal intracardiac echogenic focus to trisomy 21 at the mid-trimester sonogram in women younger than 35 years. Ultrasound in obstetrics \& gynecology: The Official Journal of the International Society of Ultrasound in Obstetrics and Gynecology 2003; 21: 354-358

[15] Bromley B, Lieberman E, Shipp TD et al. Significance of an echogenic intracardiac focus in fetuses at high and low risk for aneuploidy. Journal of Ultrasound in Medicine: Official Journal of the American Institute of Ultrasound in Medicine 1998; 17: 127-131

[16] Vibhakar NI, Budorick NE, Scioscia AL et al. Prevalence of aneuploidy with a cardiac intraventricular echogenic focus in an at-risk patient population. Journal of Ultrasound in Medicine: Official Journal of the American Institute of Ultrasound in Medicine 1999; 18: 265-268 quiz 269-270

[17] Weisz B, Pandya PP, David AL et al. Ultrasound findings after screening for down syndrome using the integrated test. Obstetrics and Gynecology 2007; 109: 1046-1052

[18] Shanks AL, Odibo AO, Gray DL. Echogenic intracardiac foci: associated with increased risk for fetal trisomy 21 or not? Journal of Ultrasound in Medicine: Official Journal of the American Institute of Ultrasound in Medicine 2009; 28: 1639-1643 
[19] Huang SY, Shaw SW, Cheuh HY et al. Intracardiac echogenic focus and trisomy 21 in a population previously evaluated by first-trimester combined screening. Acta obstetricia et gynecologica Scandinavica 2010; 89: 1017-1023

[20] Verde PE, Ohmann C. Combining randomized and non-randomized evidence in clinical research: a review of methods and applications. Research Synthesis Methods 2015; Mar 6: 45-62

[21] Agathokleous M, Chaveeva P, Poon LC et al. Meta-analysis of second-trimester markers for trisomy 21. Ultrasound in Obstetrics \& Gynecology: The Official Journal of the International Society of Ultrasound in Obstetrics and Gynecology 2013; 41: 247-261

[22] Verde PE. bamdit: An R Package for bayesian meta-analysis of diagnostic test data. Journal of Statistical Software 2018; 86: 1-32

[23] Sepulveda W, Cullen S, Nicolaidis P et al. Echogenic foci in the fetal heart: a marker of chromosomal abnormality. $\mathrm{Br}$ J Obstet Gynaecol 1995; 102: 490-492

[24] Roberts D], Genest D. Cardiac histologic pathology characteristic of trisomies 13 and 21. Hum Pathol 1992; 23: 1130-1140
[25] Lehman CD, Nyberg DA, Winter TC 3rd et al. Trisomy 13 syndrome: Prenatal US findings in a review of 33 cases. Radiology 1995; 194: 217-222

[26] Kozlowski P, Burkhardt T, Gembruch U et al. DEGUM, OGUM, SGUM and FMF Germany recommendations for the implementation of first-trimester screening, detailed ultrasound, cell-free dna screening and diagnostic procedures. Ultraschall in Med (Stuttgart, Germany: 1980) 2018; DOI: $10.1055 / \mathrm{a}-0631-8898$

[27] Revello R, Sarno L, Ispas A et al. Screening for trisomies by cell-free DNA testing of maternal blood: Consequences of a failed result. Ultrasound in obstetrics \& gynecology: The Official Journal of the International Society of Ultrasound in Obstetrics and Gynecology 2016; 47: 698-704

[28] Akolekar R, Beta J, Picciarelli G et al. Procedure-related risk of miscarriage following amniocentesis and chorionic villus sampling: A systematic review and meta-analysis. Ultrasound in Obstetrics \& Gynecology: The Official Journal of the International Society of Ultrasound in Obstetrics and Gynecology 2015; 45: 16-26 